\title{
Chiral symmetry of QCD with twelve light flavors
}

\author{
A. Deuzeman * \\ Centre for Theoretical Physics, University of Groningen, 9747 AG, Netherlands \\ E-mail: a.deuzemanerug.nl \\ M. P. Lombardo \\ INFN-Laboratori Nazionali di Frascati, I-00044, Frascati (RM), Italy \\ E-mail: lombardo@lnf.infn.it \\ E. Pallante \\ Centre for Theoretical Physics, University of Groningen, 9747 AG, Netherlands \\ E-mail: e.pallante@rug.nl
}

\begin{abstract}
We study QCD with twelve light flavors at intermediate values of the bare lattice coupling. We contrast the results for the order parameter with different theoretical models motivated by the physics of the Goldstone phase and of the symmetric phase, and we perform a model independent analysis of the meson spectrum inspired by universal properties of chiral symmetry. Our analysis favors chiral symmetry restoration.
\end{abstract}

The XXVIII International Symposium on Lattice Field Theory, Lattice2010 June 14-19, 2010

Villasimius, Italy

\footnotetext{
*Present address: Institute for Theoretical Physics, Ch-3012 Bern, Switzerland † Speaker.
} 


\section{Introduction}

In this note -which is part of an extended publication [1] - we discuss the realization of chiral symmetry in QCD with twelve flavors. This is an ongoing effort which aims at clarifying the phase diagram of QCD with fundamental fermions - effort motivated either by theoretical and phenomenological considerations, nicely reviewed in e.g. [2-4]. The salient feature to be elucidated is the occurrence of a zero temperature phase transition in QCD when the number of flavor grows large, but still below the loss of asymptotic freedom [5]. This phase transition separating the hadronic phase of QCD from a symmetric, conformal phase is a predictions from different model and analytic studies, and still needs a quantitative, first principle assessment. To briefly summarize the status of the field at the time of this meeting, there is little doubt that SU(3) theories with a number of flavors below or equal eight are in the ordinary, broken phase of QCD [6-8]. QCD with twelve flavors appears to be a borderline case, either from an analytic and numerical point of view: our results [1] as well as results based on the analysis of the Schroedinger functional [6] favor chiral symmetry restoration at weak coupling, and indicate the existence of an IRFP, others suggest chiral symmetry breaking [9]. According to a MCRG analysis [10] the data favor the existence of an infrared fixed point and conformal phase, though the results are also consistent with very slow walking.

It might be worthwhile to recall that conformality implies chiral symmetry - indeed the conformal window has been uncovered by noticing that, in the presence of an IRFP, the running coupling might not grow large enough to break chiral symmetry [11]. Only at a later stage it was recognized that the chiral transition at the critical number of flavor was of a peculiar nature - dubbed conformal [5]. Hence, chiral symmetry restoration in the asymptotically free region of QCD, and conformality go hand-in-hand. The direct observation of the physics of a conformal transition and the behaviour at IRFP are of course an important subject of investigation, which we are not addressing here.

\section{The simulations, their systematics and the bulk transition}

We have simulated an $S U(3)$ gauge theory with twelve flavors of staggered fermions in the fundamental representation. We used a tree level Symanzik improved gauge action to suppress lattice artifacts, and Kogut-Susskind (staggered) fermions with the Naik improvement scheme. High statistics runs were performed at fixed bare quark mass $a m=0.05$ over an extended range of bare lattice couplings, on $16^{3} \times 8$ and $16^{4}$ lattices. The results for the chiral condensate [1] suggest a bulk phase transition at $g_{L}=1.35(3)$.

At two selected couplings, $6 / g_{L}^{2}=3.9$ and $6 / g_{L}^{2}=4.0$, on the weak coupling side of the bulk transition, we have then performed runs on lattices $20^{3} \times 32,24^{4}, 32^{4}$ and five masses am = $0.025,0.04,0.05,0.06,0.07$. The thermalization of all runs was extensively verified by monitoring the stability of averages and uncertainties as a function of the discarded number of sweeps, and bin size. In addition, we have verified the decorrelation from initial conditions by performing simulations with ordered and random starts for a few selected couplings and masses.

Measurements of the chiral condensate were performed on three different volumes for each mass, up to $32^{4}$ for the smallest masses. The data set used for the extrapolation to the chiral limit 


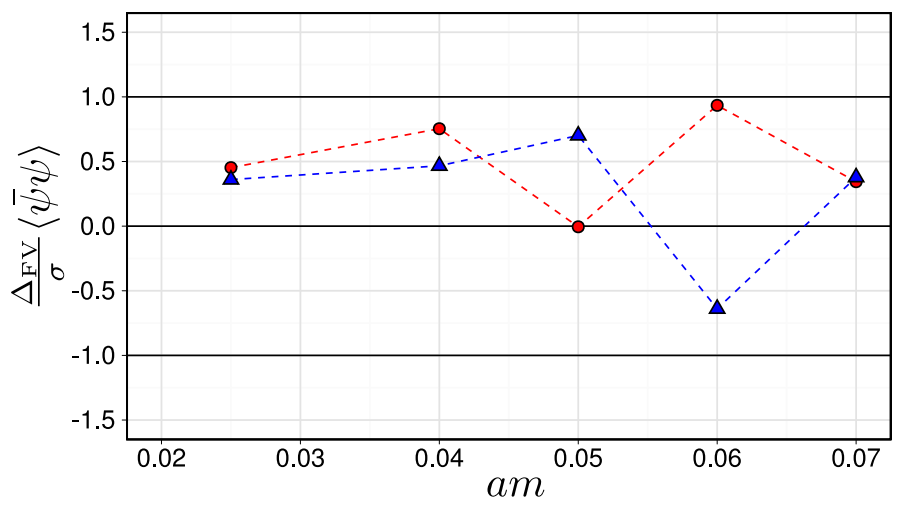

Figure 1: (color online) Observed finite volume effects in the chiral condensate, displayed as the difference $\Delta_{\mathrm{FV}}$ between the measurements at the two largest available volumes $\left(24^{3} \times 24\right.$ and $32^{3} \times 32$ for the lowest mass, $20^{3} \times 32$ and $24^{3} \times 24$ for the other masses) divided by their combined standard deviation $\sigma$. Blue triangles indicate results for $6 / g_{L}^{2}=3.9$, red circles those for $6 / g_{L}^{2}=4.0$.

consists of the measurements at lattice volumes $24^{4}$, which can be considered as infinite volume measurements within their errors, see Fig. 1.

\section{The analysis of the chiral condensate}

Having observed a behaviour strongly suggestive of a bulk phase transition, we examine in detail the chiral condensate, and the spectrum (next Section) for two selected coupling on the weak coupling side of the transition itself. Why staying close to the bulk transition? For one thing, the finite volume effects (which are expected to be large in a symmetric phase), should anyway be smaller at larger coupling. Second, close to the bulk transition the system should be QED-like and we can use previous expertise coming from the lattice analysis of the QED strong coupling transition. On a more technical note, we underscore that staggered fermions have a remnant of exact chiral symmetry which allows a precise definition of the chiral order parameter - the condensate $\langle\bar{\psi} \psi\rangle$ - also on a coarse lattice, with a genuine Goldstone modes and mass gap when the symmetry is broken.

\subsection{Fits motivated by a possible Goldstone phase}

The functional forms discussed here would be appropriate if the bulk behavior were not to be associated to a true chiral transition. For instance, it might just be due to a generic rapid crossover, or to a genuinely lattice transition between two phases with different ordering. In this case the range of couplings between $6 / g_{L}^{2}=3.9$ and $6 / g_{L}^{2}=4.0$ would still belong to the phase with broken chiral symmetry. We have thus considered the following functional form:

$$
\langle\bar{\psi} \psi\rangle=A m+B m \log (m)+\langle\bar{\psi} \psi\rangle_{0},
$$

where the parameters were all left free, giving fits with two degrees of freedom, or in turn constrained to zero. The logarithmic mass dependence is typical of a chirally broken phase for a QCD-like theory in four dimensions at zero temperature. 


\begin{tabular}{|c|c|c|c|c|}
$6 / g_{L}^{2}$ & $\mathrm{~A}$ & $\mathrm{~B}$ & $\langle\bar{\psi} \psi\rangle_{0}$ & $\sqrt{\chi^{2} \mathrm{dof}}$ \\
\hline 3.9 & $2.70(3)$ & $-0.103(13)$ & $0.00013(54)$ & 0.68 \\
& $3.12(3)$ & $0(\mathrm{~F})$ & $0.0043(3)$ & 3.12 \\
& $2.682(5)$ & $-0.107(2)$ & $0(\mathrm{~F})$ & 0.56 \\
\hline 4.0 & $2.48(2)$ & $-0.120(10)$ & $-0.00091(42)$ & 0.51 \\
& $2.73(1)$ & $0(\mathrm{~F})$ & $0.0041(5)$ & 3.74 \\
& $2.519(8)$ & $-0.099(3)$ & $0(\mathrm{~F})$ & 0.56
\end{tabular}

Table 1: Fits to $\langle\bar{\psi} \psi\rangle=A m+B m \log m+\langle\bar{\psi} \psi\rangle_{0}$

The results of the fits to Eq. 3.1 are summarized in Table 1. The linear fits produce an intercept different from zero, but are highly disfavored by their large $\chi^{2}$. The inclusion of the term $m \log (m)$ considerably improves the quality of the fits. Those with free intercept $\langle\bar{\psi} \psi\rangle_{0}$ gave an extrapolated value consistent with zero, and in agreement with the fit obtained by constraining $\langle\bar{\psi} \psi\rangle_{0}=0$. Both fits are satisfactory, and imply that the chiral condensate in the chiral limit is zero within errors. In conclusion, a conventional picture of the Goldstone phase seems not to be supported by our data.

\subsection{Fits with an anomalous dimension}

We considered the functional form

$$
\langle\bar{\psi} \psi\rangle=A m^{1 / \delta}+B m+\langle\bar{\psi} \psi\rangle_{0}
$$

containing an anomalous dimension, whose effect is parameterized by the exponent $\delta$. Since the fits described in section 3.1 already suggest that a curvature in the behavior of the chiral condensate as a function of the mass is mandatory, we started by setting the linear term to zero. We note that analogous fits were used in the past to analyze QED in its symmetric phase, close to the strong coupling transition in Ref. [12], even if a more satisfactory account of the data requires the consideration of the magnetic equation of state, which is going to be discussed in the next section. All fits to Eq. (3.2) with $B=0$ are satisfactory, with a chiral condensate compatible with zero in the chiral limit. This was checked, as before, by comparing fits with free intercept, and fits with $\langle\bar{\psi} \psi\rangle_{0}=0$.

One might still suspect that a fit combining a power-law term and a linear term, with a non zero intercept might still accommodate the data, hence indicating chiral symmetry breaking. For completeness we have performed fits to Eq. (3.2) with the inclusion of a linear term. As expected from the near degeneracy between a power law with $1 / \delta \approx 1$ and a linear term, the uncertainties coming from a Marquardt-Levenberg minimization of $\chi^{2}$ are huge.

To acquire a feeling about the possible relevance of a linear term, we have also performed a sequence of fits, constraining the exponent to several values in the acceptable range given by the fit errors. It appears that the coefficient of the linear term smoothly changes from positive to negative, while the intercept - the chiral condensate in the chiral limit - remains consistent with zero throughout at $6 / g_{L}^{2}=3.9$, and becomes slightly negative at $6 / g_{L}^{2}=4.0$. We thus again conclude that our data point at exact chiral symmetry. 


\begin{tabular}{|c|c|c|c|}
$6 / g_{L}^{2}$ & $\mathrm{~A}$ & $\mathrm{~B}$ & $\delta$ \\
\hline 3.9 & $0.1(9)$ & $0.3(9)$ & $1.1(2)$ \\
\hline 4.0 & $0.3(1)$ & $0.077(9)$ & $1.3(1)$
\end{tabular}

Table 2: Fits to $m=A\langle\bar{\psi} \psi\rangle+B\langle\bar{\psi} \psi\rangle^{\delta}$

In conclusion, regardless the presence of any additional linear term, or any analytic term in Eq. (3.2) the resulting extrapolated condensate remains compatible with zero. The interested reader is referred again to our extended publication Ref. [1] for details.

\subsection{Fits motivated by the Magnetic Equation of State}

Finally we considered fits motivated by the magnetic equation of state, see e.g. Ref. [12] and references therein. The following equation is a satisfactory parameterization

$$
m=A\langle\bar{\psi} \psi\rangle+B\langle\bar{\psi} \psi\rangle^{\delta},
$$

which would of course coincide with the simple power law when $\mathrm{A}=0$. The coefficient of the linear term $A$ should vanish at a critical point, with $A \propto\left(\beta-\beta_{c}\right)$. This of course explains the smallness of A close to the transition, while $\delta$ is the conventional magnetic exponent. The linear term in the condensate is implied by chiral symmetry, and guarantees that the ratio

$$
\lim _{m \rightarrow 0} R_{\pi}=\frac{\partial\langle\bar{\psi} \psi\rangle / \partial m}{\langle\bar{\psi} \psi\rangle / m}=1
$$

approaches unity in the chiral limit and in the chirally symmetric phase. We can view Eq. (3.3) as a model for a theory with anomalous dimensions, which incorporates the correct chiral limit. Note that the linear term of Eq. (3.3) is of different origin than the one considered in Eq. (3.2). The latter describes violations of scaling and it is increasingly relevant at larger masses. In Eq. (3.3) instead, it is dominating at very small masses, away from the critical point.

Results for this case are given in Table 2. The fit $m=m(\langle\bar{\psi} \psi\rangle)$ was performed with a least squares algorithm. Note that, as expected, the significance of the linear term is very low, closer to the bulk transition, and slightly larger by moving away from it. Indeed, the agreeement between the numerical solutions of the equation $m(\langle\bar{\psi} \psi\rangle)=m_{\text {sim }}$, with $m_{\text {sim }}$ the simulation masses, with the simulation results for the condensate is very good.

All fits clearly favor a positive value for the coefficient of the linear term, as it should be in the chirally symmetric phase, and within the large errors the results for the exponent are compatible with the ones coming from the genuine power law fits. We conclude again in favor of chiral symmetry restoration.

\section{Spectrum analysis}

We have fitted both the pion and the rho mass to a power law

$$
m_{\pi, \rho}=A_{\pi, \rho} m^{\varepsilon_{\pi, \rho}}
$$




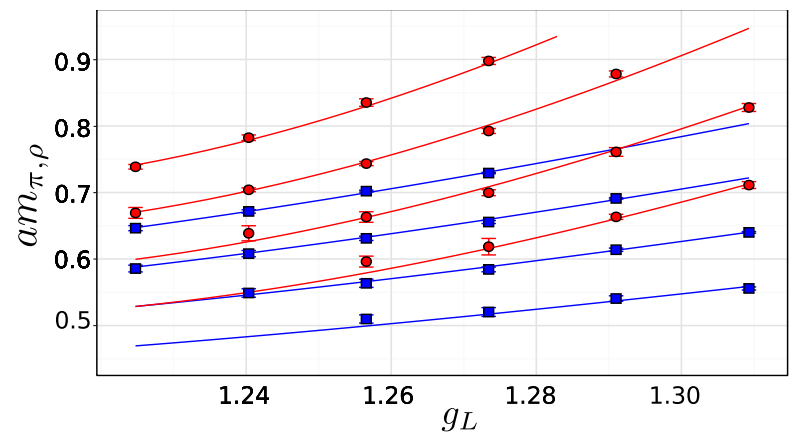

(a) Interpolations of meson masses.

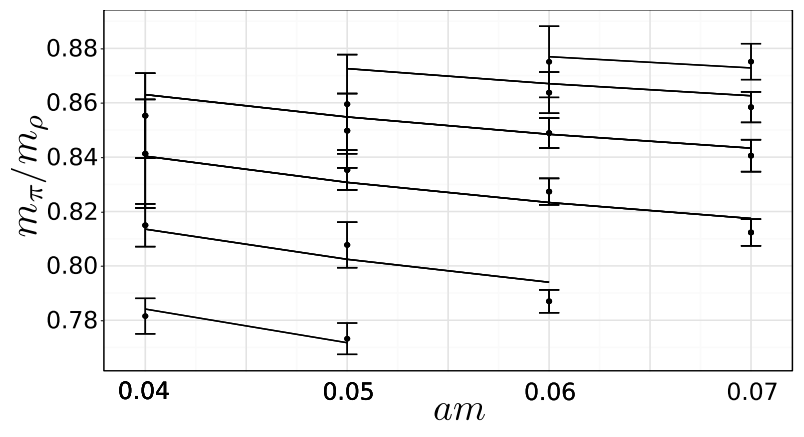

(b) $\pi$ to $\rho$ mass ratio with fits superimposed

Figure 2: (color online) (a) Measurements of the pseudoscalar (blue) and vector (red) masses versus lattice coupling at several values of the bare quark mass, from bottom to top am $=0.04,0.05,0.06$ and 0.07 , with fits superimposed. (b) The measured $\pi$ to $\rho$ mass ratio as a function of the bare mass and decreasing coupling $g_{L}$, bottom to top $6 / g_{L}^{2}=3.5$ to 4 . The superimposed lines are ratios of the best fits.

with the results $A_{\pi}=3.41(21), \varepsilon_{\pi}=0.61(2), A_{\rho}=4.47(61), \varepsilon_{\rho}=0.66(5)$ at $6 / g_{L}^{2}=3.9$, and $A_{\pi}=3.41(21), \varepsilon_{\pi}=0.61(2), A_{\rho}=4.29(11), \varepsilon_{\rho}=0.66(1)$ at $6 / g_{L}^{2}=4.0$. From these fits - also plotted in Fig. 2(a) - we conclude that the mass dependence of the vector and pseudoscalar mesons is well fitted by a power-law. Second, it is also relevant that the exponents are not unity and $\varepsilon_{\pi} \neq 1 / 2$. The latter result immediately tells that the pion seen here is not a Goldstone boson of a broken chiral symmetry.

By looking at the behavior of the mass ratio we can further inspect the status of chiral symmetry. Fig. 2(b) shows the ratios of measured pseudoscalar and vector masses, for a fixed coupling and as a function of the bare quark mass. We have superimposed the ratios of the best fits to the raw mass data. It is immediately clear that the ratio increases as the quark mass approaches zero, a behavior opposite to what is expected for a Goldstone pion (see Ref. [12] for more exteded discussions) .

Note that the spectrum results can be used to determine the lines of "constant physics" in the two dimensional parameter space $g_{L}$ and $a m$, the bare quark mass of degenerate fermions, following the same strategy which was successful for $N_{f}=16$ [13].

\section{Summary and Outlook}

After observing a bulk transition for $N_{f}=12$, we are continuing our study along two lines: we explored the nature of the weak coupling phase, which is the subject of this note, and we are investigating in more details the nature of this bulk transition, see the contribution by Elisabetta Pallante [14].

The results presented here favor chiral symmetry restoration in the range of couplings which we have explored. Barring unexpected re-entrant transitions at even weaker coupling (a preliminary $\beta$ scan shows no sign of such phase transitions) this should indicate chiral symmetry also in the continuum limit, compatible with the existence of an IRFP.

Can we challenge this result? It is clear that additional data at even lighter masses or extremely larger volumes might in principle leave room for different conclusions. In practice, it seems difficult 
to reverse the trend of the mass ratio.

If the $N_{f}=12$ theory is very close to the critical number of flavor it is indeed rather natural to have ambiguous results from different methods and setups. The analysis of the phase diagram in the Temperature- $N_{f}$ space [15] might help understanding why: if the critical exponent of the running gauge coupling at the IRFP is less then one, the critical temperature becomes exponentially small when approaching $N_{f}^{c}$ : a cold quark gluon plasma will then become arbitrarily close to the zero temperature conformal window, and the hadronic phase will only appear for a very large $N_{t}$.

A conservative conclusion - encompassing our results as well as those from other groups - is then that the critical number of flavor is very close to twelve, and a speculation is that the critical exponent of the running gauge coupling at the Banks-Zacs fixed point is less than one.

\section{References}

[1] A. Deuzeman, M. P. Lombardo, and E. Pallante, Evidence for a conformal phase in SU(N) gauge theories, Phys. Rev. D82 (2010) 074503, [arXiv: 0904 .4662].

[2] F. Sannino, Phase Diagrams of Strongly Interacting Theories, arXiv:1003.0289.

[3] L. Del Debbio, , This Volume.

[4] T. DeGrand, Lattice studies of QCD-like theories with many fermionic degrees of freedom, arXiv: 1010.4741.

[5] V. A. Miransky and K. Yamawaki, Conformal phase transition in gauge theories, Phys. Rev. D 55 (Apr., 1997) 5051.

[6] T. Appelquist, G. T. Fleming, and E. T. Neil, Lattice Study of the Conformal Window in QCD-like Theories, Phys. Rev. Lett. 100 (2008) 171607, [arXiv:0712. 0609 ].

[7] A. Deuzeman, M. P. Lombardo, and E. Pallante, The physics of eight flavours, Phys. Lett. B670 (2008) 41-48, [arXiv:0804.2905].

[8] X.-Y. Jin and R. D. Mawhinney, Lattice QCD with Eight Degenerate Quark Flavors, PoS LATTICE2008 (2008) 059, [arXiv: 0812 . 0413 ].

[9] Z. Fodor, K. Holland, J. Kuti, D. Nogradi, and C. Schroeder, Nearly conformal gauge theories in finite volume, Phys. Lett. B681 (2009) 353-361, [arXiv : 0907 . 4562].

[10] A. Hasenfratz, Conformal or Walking? Monte Carlo renormalization group studies of SU(3) gauge models with fundamental fermions, Phys. Rev. D82 (2010) 014506, [arXiv: 1004.1004 ].

[11] T. Appelquist, J. Terning, and L. Wijewardhana, The zero temperature chiral phase transition in su(n) gauge theories, Phys. Rev. Lett. 77 (1996) 1214-1217.

[12] A. Kocic, S. Hands, J. B. Kogut, and E. Dagotto, The equation of state and critical exponents in quenched strongly coupled QED, Nucl. Phys. B347 (1990) 217-242.

[13] P. Damgaard, U. Heller, A. Krasnitz, and P. Olesen, Lattice QCD with many flavours, Phys. Lett. B 400 (May, 1997) 169-175.

[14] A. Deuzeman, M. P. Lombardo, and E. Pallante, talk presented by E. Pallante, This Volume.

[15] J. Braun and H. Gies, Scaling laws near the conformal window of many-flavor QCD, JHEP 05 (2010) 060, [arXiv:0912.4168]. 